\title{
A forwarding strategy for DWSIGF routing protocol
}

\begin{abstract}
Routing protocols in Wireless Sensor Network (WSN) are responsible for propagating and coordinating of information transfer from one end of the network to the other. Dynamic Window Secured Implicit Geographic Forwarding (DWSIGF) is a robust, cross layer, security bound routing protocol that propagates information in a multi-hop network using the greedy and random forwarding strategies. These strategies are known for their poor resistivity to interference and erratic behavior in path selection. In this paper, we propose a forwarding strategy that uses an optimal distance to mitigate these problems. The optimal distance is computed based on the path loss coefficient and energy dissipated in the hardware (sensor). Extensive simulations have been conducted to evaluate the performance of the proposed approach. The results illustrate that the proposed approach performs better than the compared strategies in terms of packet delivery ratio and energy consumption.
\end{abstract}

Keyword: Energy consumption; Hardware; Routing; Routing protocols; Security; Wireless sensor networks 